\title{
ON GROUPS ACTING ON CONTRACTIBLE SPACES WITH STABILIZERS OF PRIME POWER ORDER
}

\author{
IAN J. LEARY AND BRITA E. A. NUCINKIS
}

\begin{abstract}
Let $\mathfrak{F}$ denote the class of finite groups, and let $\mathfrak{P}$ denote the subclass consisting of groups of prime power order. We study group actions on topological spaces in which either (1) all stabilizers lie in $\mathfrak{P}$ or $(2)$ all stabilizers lie in $\mathfrak{F}$. We compare the classifying spaces for actions with stabilizers in $\mathfrak{F}$ and $\mathfrak{P}$, the Kropholler hierarchies built on $\mathfrak{F}$ and $\mathfrak{P}$, and group cohomology relative to $\mathfrak{F}$ and to $\mathfrak{P}$. In terms of standard notations, we show that $\mathfrak{F} \subset \mathbf{H}_{1} \mathfrak{P} \subset \mathbf{H}_{1} \mathfrak{F}$, with all inclusions proper; that $\mathbf{H} \mathfrak{F}=\mathbf{H} \mathfrak{P}$; that $\mathfrak{F} H^{*}(G ;-)=\mathfrak{P} H^{*}(G ;-)$; and that $\mathrm{E}_{\mathfrak{P}} G$ is finite-dimensional if and only if $\mathrm{E}_{\mathfrak{F}} G$ is finite-dimensional and every finite subgroup of $G$ is in $\mathfrak{P}$.
\end{abstract}

\section{INTRODUCTION}

Let $\mathcal{F}$ denote a family of subgroups of a group $G$, by which we mean a collection of subgroups which is closed under conjugation and inclusion. A $G$-CW-complex $X$ is said to be a model for $\mathrm{E}_{\mathcal{F}} G$, the classifying space for actions of $G$ with stabilizers in $\mathcal{F}$, if the fixed point set $X^{H}$ is contractible for $H \in \mathcal{F}$ and is empty for $H \notin \mathcal{F}$. The most common families considered are the family consisting of just the trivial group and the family $\mathfrak{F}$ consisting of all finite subgroups of $G$. In these cases $\mathrm{E}_{\mathcal{F}} G$ is often denoted $\mathrm{E} G$ and $\underline{\mathrm{E}} G$ respectively. Note that $\mathrm{E} G$ is the total space of the universal principal $G$-bundle, or equivalently the universal covering space of an Eilenberg-Mac Lane space for $G$. The space $\underline{E} G$ is called the classifying space for proper actions of $G$. Recently there has been much interest in finiteness conditions for classifying spaces for families, especially for $\underline{E} G$. Milnor and Segal's constructions of $\mathrm{E} G$ both generalize easily to construct models for any $\mathrm{E}_{\mathcal{F}} G$, and one can show that any two models for $\mathrm{E}_{\mathcal{F}} G$ are naturally equivariantly homotopy equivalent.

For some purposes the structure of the fixed point sets for subgroups in $\mathcal{F}$ is irrelevant. For example, a group is in Kropholler's class $\mathbf{H}_{1} \mathcal{F}$

The first named author was partially supported by NSF grant DMS-0804226 and by the Heilbronn Institute. 
if there is any finite-dimensional contractible $G$-CW-complex $X$ with all stabilizers in $\mathcal{F}$. The class $\mathbf{H}_{1} \mathcal{F}$ is the first stage of a hierarchy whose union is Kropholler's class $\mathbf{H} \mathcal{F}$ of hierarchically decomposable groups [8]. (These definitions were first considered for the class $\mathfrak{F}$ of all finite groups, but work for any family $\mathcal{F}$.)

A priori, the class $\mathbf{H}_{1} \mathcal{F}$ may contain groups $G$ that do not admit a finite-dimensional model for $\mathrm{E}_{\mathcal{F}} G$, and we shall give such examples in the case when $\mathcal{F}=\mathfrak{P}$, the class of groups of prime power order. By contrast, in the case when $\mathcal{F}=\mathfrak{F}$, no group $G$ is known to lie in $\mathbf{H}_{1} \mathfrak{F}$ without also admitting a finite-dimensional model for $\underline{E} G$. A construction due to Serre shows that every group $G$ in $\mathbf{H}_{1} \mathfrak{F}$ that is virtually torsion-free has a finite-dimensional $\underline{\mathrm{E}} G$ [4], and the authors have given examples of $G$ for which the minimal dimension of a contractible $G$-CW-complex is lower than the minimal dimension of a model for EG [12]. These examples $G$ also have the property that they admit a contractible $G$-CW-complex with finitely many orbits of cells, but that they do not admit any model for $\underline{E} G$ with finitely many orbits of cells.

Throughout this paper, $\mathfrak{F}$ will denote the family of finite groups, and $\mathfrak{P}$ will denote the family of finite groups of prime power order. We compare the classifying space for $G$-actions with stabilizers in $\mathfrak{P}$ with the more well-known $\underline{\mathrm{E}} G$, and we compare the Kropholler hierarchies built on $\mathfrak{F}$ and $\mathfrak{P}$. We show that a finite group $G$ that is not of prime power order cannot admit a finite-dimensional $\mathrm{E}_{\mathfrak{P}} G$, but that every finite group is in $\mathbf{H}_{1} \mathfrak{P}$. We also construct a group that is in $\mathbf{H}_{1} \mathfrak{F}$ but not in $\mathbf{H}_{1} \mathfrak{P}$, and we show that $\mathbf{H} \mathfrak{P}=\mathbf{H} \mathfrak{F}$.

In the final section we shall contrast this with cohomology relative to the family of all finite subgroups. The relative cohomological dimension can be viewed as a generalisation of the virtual cohomological dimension, since for virtually torsion free groups these are equal, see [15]. By a result of Bouc $[2,10]$ it follows that groups belonging to $\mathbf{H}_{1} \mathfrak{F}$ have finite relative cohomological dimension, but the converse it not known. In contrast to our results concerning classifying spaces for families, we show that cohomology relative to subgroups in $\mathfrak{F}$ is naturally isomorphic to cohomology relative to subgroups in $\mathfrak{P}$.

\section{Classifying spaces for the family of $\mathfrak{P}$-Subgroups}

Proposition 2.1. Let $G$ be a finite group. Then $G$ has a finite dimensional model for $\mathrm{E}_{\mathfrak{P}} G$ if and only if $G$ has prime power order.

Proof. If $G$ has prime power order, then a single point may be taken as a model for $\mathrm{E}_{\mathfrak{P}} G$. Now let $G$ be an arbitrary finite group, let $p$ be a 
prime dividing the order of $G$, and assume that there is a $p$-subgroup $P<G$, such that $N_{G}(P)$ is not a $p$-group. Then the Weyl-group $W P=N_{G}(P) / P$ contains a subgroup $H$ of order prime to $p$. Assume $G$ has a finite dimensional model for $\mathrm{E}_{\mathfrak{P}} G, X$ say. Then the augmented cellular chain complex of the $P$-fixed point set, $X^{P}$, is a finite length resolution of $\mathbb{Z}$ by free $H$-modules. This gives a contradiction, since $\mathbb{Z}$ has infinite projective dimension as an $H$-module for any non-trivial finite group $H$.

Therefore we may suppose that $G$ is not in $\mathfrak{P}$ and for all non-trivial subgroups $P \in \mathfrak{P}$, the normalizer $N_{G}(P)$ is also in $\mathfrak{P}$. Now let $N$ be a minimal normal subgroup of $G$. This cannot lie in $\mathfrak{P}$ and hence has the same properties as $G$. Thus, by minimality we may assume $N=G$ and $G$ is simple.

Choose two distinct Sylow $p$-subgroups $P$ and $Q$ of $G$, such that their intersection, $I$ say, is of maximal order. Now, the normalisers $N_{P}(I)$ and $N_{Q}(I)$ contain $I$ as a proper subgroup. Also, the group $\left\langle N_{P}(I), N_{Q}(I)\right\rangle$ does not contain $P$ and $Q$ and neither does $N_{G}(I) \geq$ $\left\langle N_{P}(I), N_{Q}(I)\right\rangle$, which is a $p$-group by assumption. Hence there exists a Sylow $p$-subgroup $R$ containing $N_{G}(I)$ and $R \cap P \geq N_{P}(I)$. Thus $\mid R \cap$ $P|>| I|=| P \cap Q \mid$, which contradicts the maximality of $I$. Therefore we may assume that in $G$ all Sylow $p$-subgroups intersect trivially. In such a group we have, for $P$ a Sylow $p$-subgroup:

$$
H^{*}\left(G, \mathbb{F}_{p}\right) \cong H^{*}\left(P, \mathbb{F}_{p}\right),
$$

see for example [4, Theorem III.10.3].

Any non-trivial $p$-group has non-trivial abelianization, and hence $H^{1}\left(P, \mathbb{F}_{p}\right)$, which is naturally isomorphic to $\operatorname{Hom}\left(P, \mathbb{F}_{p}\right)$, is non-trivial. But this implies that $H^{1}\left(G, \mathbb{F}_{p}\right) \cong \operatorname{Hom}\left(G, \mathbb{F}_{p}\right)$ is non-trivial, and so $G$ admits a surjective homomorphism to a group of order $p$. Since $G$ is not in $\mathfrak{P}$, it follows that $G$ cannot be simple, which gives the final contradiction.

Corollary 2.2. For a group $G$, the following are equivalent.

(i) $G$ admits a finite-dimensional $\mathrm{E}_{\mathfrak{P}} G$;

(ii) Every finite subgroup of $G$ is in $\mathfrak{P}$ and $G$ admits a finitedimensional $\mathrm{E} G$.

Remark 2.3. We conclude the section with a remark on the type of $\mathrm{E}_{\mathfrak{P}} G$. It can proved analogously to Lück's proof for $\underline{\mathrm{E}} G$ [13] that a group $G$ admits a finite type model for $\mathrm{E}_{\mathfrak{P}} G$ if and only if $G$ has finitely many conjugacy classes of groups of prime power order and the Weyl-groups $N_{G}(P) / P$ for all subgroups $P$ of prime power order are finitely presented and of type $\mathrm{FP}_{\infty}$. Hence any group admitting a finite 
type $\mathrm{E} G$ also admits a finite type $\mathrm{E}_{\mathfrak{P}} G$. Recall that a finite extension of a group admitting a finite model for $\mathrm{E} G$ always has finitely many conjugacy classes of subgroups of prime power order [4, IX.13.2]. Hence the groups exhibited in [12, Example 7.4] are groups admitting a finite type $\mathrm{E}_{\mathfrak{P}} G$ which do not admit a finite type $\underline{\mathrm{E}} G$.

This behaviour is in stark contrast to that of $\mathrm{E}_{\mathcal{V C}} G$, the classifying space with virtually cyclic isotropy. Any group admitting a finite dimensional model for $\mathrm{E}_{\mathcal{V C}} G$ admits a finite dimensional model for $\mathrm{E} G$, see [14] and the converse also holds for a large class of groups including all polycyclic-by-finite and all hyperbolic groups [6, 14]. Furthermore, any group admitting a finite type model for $\mathrm{E}_{\mathcal{V C}} G$ also admits a finite type model for $\underline{\mathrm{E}} G$ [7], but it is conjectured [6] that any group admitting a finite model for $\mathrm{E}_{\mathcal{V C}} G$ has to be virtually cyclic. This has been shown for a class of groups containing all hyperbolic groups [6] and for elementary amenable groups [7].

\section{THE HIERARCHIES $\mathbf{H} \mathfrak{F}$ AND $\mathbf{H} \mathfrak{P}$}

Proposition 3.1. Let $X$ be a finite dimensional contractible $G-C W$ complex such that all stabilizers are finite. If there is a bound on the orders of the stabilizers then there exists a finite dimensional contractible $G$-CW-complex $Y$ and an equivariant map $f: Y \rightarrow X$ such that $Y^{H}=\varnothing$ if $H$ is not a p-group.

Proof. Using the equivariant form of the simplicial approximation theorem, we may assume that $X$ is a simplicial $G$-CW-complex. To simplify notation the phrase ' $G$-space' shall mean 'simplicial $G$-CW-complex' and ' $G$-map' will mean ' $G$-equivariant simplicial map' throughout the rest of the proof. The space $Y$ will be a $G$-space in this sense and the map $f: Y \rightarrow X$ will be a $G$-map in this sense. The $G$-space $Y$ is constructed in two stages. Firstly, for each finite $H \leq G$ we build a finite-dimensional contractible $H$-space $Y_{H}$ with the property that all simplex stabilizers in $Y_{H}$ lie in $\mathfrak{P}$.

Suppose for now that each such $H$-space $Y_{H}$ has been constructed. Using the $G$-equivariant form of the construction used in $[9$, Section $8]$ the space $Y$ is constructed as follows. Let $I$ be an indexing set for the $G$-orbits of vertices in $X$. For each $i \in I$, let $v_{i}$ be a representative of the corresponding orbit, and let $H_{i}$ be the stabilizer of $v_{i}$. Let $X^{0}$ denote the 0 -skeleton of $X$. Define a $G$-space $Y^{0}$ by

$$
Y^{0}=\coprod_{i \in I} G \times_{H_{i}} Y_{H_{i}}
$$


and define a $G$-map $f: Y^{0} \rightarrow X^{0}$ by $f(g, y)=g . v_{i}$ for all $i \in I$, for all $g \in G$ and for all $y \in Y_{H_{i}}$. For each vertex $w$ of $X$, let $Y(w)=$ $f^{-1}(w) \subset Y^{0}$. Each $Y(w)$ is a contractible subspace of $Y^{0}$, and the stabilizer of $w$ acts on $Y(w)$.

Now for $\sigma=\left(w_{0}, \ldots, w_{n}\right)$ an $n$-simplex of $X$, define a space $Y(\sigma)$ as the join

$$
Y(\sigma)=Y\left(w_{0}\right) * Y\left(w_{1}\right) * \cdots * Y\left(w_{n}\right) .
$$

Each vertex of $Y(\sigma)$ is already a vertex of one of the $Y\left(w_{i}\right)$, and so the map $f: Y^{0} \rightarrow X^{0}$ defines a unique simplicial map $f: Y(\sigma) \rightarrow \sigma$. By construction, whenever $\tau$ is a face of $\sigma$, the space $Y(\tau)$ is identified with a subspace of $Y(\sigma)$. This allows us to define $Y$ and $f: Y \rightarrow X$ as the colimit over the simplices $\sigma$ of $X$ of the subspaces $Y(\sigma)$, and to define $f: Y \rightarrow X$, which is a $G$-map of $G$-spaces. Since each $Y(\sigma)$ is contractible, it follows that $f$ is a homotopy equivalence, and hence $Y$ is also contractible (see [9, Corollary 8.6]).

It remains to build the $H$-space $Y_{H}$ for each finite group $H<G$. In the case when $H \in \mathfrak{P}$ we may take a single point to be $Y_{H}$, and so we may suppose that $H \notin \mathfrak{P}$. Fix such a subgroup $H$, and suppose that we are able to construct a finite-dimensional contractible $H$-space $Z_{H}$ in which each stabilizer is a proper subgroup of $H$. We may assume by induction that for each $K<H$ we have already constructed the $K$-space $Y_{K}$. The $H$-space $Y_{H}$ can now be constructed from $Z_{H}$ and the spaces $Y_{K}$ using a process similar to the construction of $Y$ from $X$ and the spaces $Y_{H}$. It remains to construct the $H$-space $Z_{H}$.

An explicit construction of an $H$-space $Z_{H}$ with the required properties is given in [11]. We therefore provide only a sketch of the argument. We may assume that $H$ is not in $\mathfrak{P}$. Let $S$ be the unit sphere in the reduced regular complex representation of $H$, so that $S$ is a topological space with $H$-action such that the stabilizer of every point of $S$ is a proper subgroup of $H$. Since $H$ is not in $\mathfrak{P}$, there are $H$-orbits in $S$ of coprime lengths. Using this property, it can be shown that the sphere $S$ admits an $H$-equivariant self-map $g: S \rightarrow S$ of degree zero. The $H$-space $Z_{H}$ is defined to be the infinite mapping telescope (suitably triangulated) of the map $g$.

Corollary 3.2. If $G$ is in $\mathbf{H}_{1} \mathfrak{F}$ and there is a bound on the orders of the finite subgroups of $G$, then $G$ is in $\mathbf{H}_{1} \mathfrak{P}$.

Remark 3.3. In Proposition 3.1, the bound on the orders of the stabilizers of $X$ is used only to give a bound on the dimensions of the spaces $Y_{H}$. In Corollary 3.8 we shall show that $\mathbf{H}_{1} \mathfrak{F} \neq \mathbf{H}_{1} \mathfrak{P}$. 
Remark 3.4. The construction in Proposition 3.1 does not preserve cocompactness, because for most finite groups $H$, the space $Y_{H}$ used in the construction cannot be chosen to be finite. A result similar to Proposition 3.1 but preserving cocompactness can be obtained by replacing $\mathfrak{P}$ by a larger class $\mathfrak{O}$ of groups. Here $\mathfrak{O}$ is defined to be the class of $\mathfrak{P}$-by-cyclic-by-P-groups. A theorem of Oliver [18] implies that any finite group $H$ that is not in $\mathfrak{O}$ admits a finite contractible $H$-CW-complex $Z_{H}^{\prime}$ in which all stabilizers are proper subgroups of $H$. Applying the same argument as in the proof of Proposition 3.1, one can show that given any contractible $G$-CW-complex $X$ with all stabilizers in $\mathfrak{F}$, there is a contractible $G$-CW-complex $Y^{\prime}$ with all stabilizers in $\mathfrak{D}$ and a proper equivariant map $f^{\prime}: Y^{\prime} \rightarrow X$. (By proper, we mean that the inverse image of any compact subset of $X$ is compact.)

For $X$ a $G$-CW-complex with stabilizers in $\mathfrak{F}$, and $p$ a prime, let $X_{\operatorname{sing}(p)}$ denote the subcomplex consisting of points whose stabilizer has order divisible by $p$. For $G$ a group and $p$ a prime, let $S_{p}(G)$ denote the poset of non-trivial finite $p$-subgroups of $G$.

Proposition 3.5. Suppose that $X$ is a finite-dimensional contractible $G$-CW-complex with all stabilizers in $\mathfrak{P}$. For each prime $p$, the mod-p homology of $X_{\operatorname{sing}(p)}$ is isomorphic to the mod-p homology of the (realization of the) poset $S_{p}(G)$.

Proof. Fix a prime $p$, and to simplify notation let $S$ denote the realization of the poset $S_{p}(G)$. For $P$ a non-trivial $p$-subgroup of $G$, let $X^{P}$ denote the points fixed by $P$, and let $S_{\geq P}$ denote the realization of the subposet of $S_{p}(G)$ consisting of all $p$-subgroups that contain $P$. By the P. A. Smith theorem [3], each $X^{P}$ is mod- $p$ acyclic. Each $S_{\geq P}$ is contractible since it is equal to a cone with apex $P$. Let $P$ and $Q$ be $p$-subgroups of $G$, and let $R=\langle P, Q\rangle$, the subgroup of $G$ generated by $P$ and $Q$. If $R$ is a $p$-group then $X^{P} \cap X^{Q}=X^{R}$, and otherwise $X^{P} \cap X^{Q}$ is empty. Similarly, $S_{\geq P} \cap S_{\geq Q}=S_{\geq R}$ if $R$ is a $p$-group and $S_{\geq P} \cap S_{\geq Q}$ is empty if $R$ is not a $p$-group.

Since each $X^{P}$ is mod- $p$ acyclic, the mod- $p$ homology $H_{*}\left(X_{\operatorname{sing}(p)}\right)$ is isomorphic to the mod- $p$ homology of the nerve of the covering $X_{\operatorname{sing}(p)}=\bigcup_{P} X^{P}$. Similarly, the mod- $p$ homology $H_{*}\left(S_{p}(G)\right)$ is isomorphic to the mod- $p$ homology of the nerve of the covering $S_{p}(G)=$ $\bigcup_{P} S_{\geq P}$. By the remarks in the first paragraph, these two nerves are isomorphic.

Proposition 3.6. Let $k$ be a finite field, and let $G$ be the group of $k$ points of a reductive algebraic group over $k$ of $k$-rank $n$. (For example, 
$G=S L_{n+1}(k)$.) Any finite-dimensional contractible $G$-CW-complex with stabilizers in $\mathfrak{P}$ has dimension at least $n$.

Proof. The hypotheses on $G$ imply that $G$ acts on a spherical building $\Delta$ of dimension $n-1$ [1, 5 , Appendix on algebraic groups]. Any such building is homotopy equivalent to a wedge of $(n-1)$-spheres. Quillen has shown that $\Delta$ is homotopy equivalent to the realization of $S_{p}(G)$, where $p$ is the characteristic of the field $k$ [19, Proposition 2.1 and Theorem 3.1]. It follows that $S_{p}(G)$ is homotopy equivalent to a wedge of $(n-1)$-spheres, and in particular the mod-p homology group $H_{n-1}\left(S_{p}(G)\right)$ is non-zero.

Now suppose that $X$ is a finite-dimensional contractible $G$-CWcomplex with stabilizers in $\mathfrak{P}$. Using Proposition 3.5 , one sees that the mod- $p$ homology group $H_{n-1}\left(X_{\operatorname{sing}(p)}\right)$ is non-zero. It follows that $X$ must have dimension at least $n$.

Remark 3.7. In [11], it is shown that in the case when $G=S L_{n+1}\left(\mathbb{F}_{p}\right)$, every contractible $G$-CW-complex without a global fixed point has dimension at least $n$.

Corollary 3.8. There are the following strict containments and equalities between classes of groups:

(i) $\mathfrak{F} \subsetneq \mathbf{H}_{1} \mathfrak{P}$;

(ii) $\mathbf{H}_{1} \mathfrak{P} \subsetneq \mathbf{H}_{1} \mathfrak{F}$;

(iii) $\mathbf{H} \mathfrak{F}=\mathbf{H} \mathfrak{P}$.

Proof. Corollary 3.2 shows that $\mathfrak{F} \subseteq \mathbf{H}_{1} \mathfrak{P}$. The free product of two cyclic groups of prime order is in $\mathbf{H}_{1} \mathfrak{P}$ and is not finite. The claim that $\mathbf{H} \mathfrak{F}=\mathbf{H P}$ follows from the inequalities $\mathfrak{P} \subseteq \mathfrak{F} \subseteq \mathbf{H}_{1} \mathfrak{P}$, and the claim $\mathbf{H}_{1} \mathfrak{P} \subseteq \mathbf{H}_{1} \mathfrak{F}$ follows from $\mathfrak{P} \subseteq \mathfrak{F}$.

It remains to exhibit a group $G$ that is in $\mathbf{H}_{1} \mathfrak{F}$ but not in $\mathbf{H}_{1} \mathfrak{P}$. Let $G=S L_{\infty}\left(\mathbb{F}_{p}\right)$, the direct limit of the groups $G_{n}=S L_{n}\left(\mathbb{F}_{p}\right)$, where $G_{n}$ is included in $G_{n+1}$ as the 'top corner'. As a countable locally-finite group, $G$ acts with finite stabilizers on a tree. (Explicitly, the vertex set $V$ and edge set $E$ are both equal as $G$-sets to the disjoint union of the sets of cosets $G / G_{1} \cup G / G_{2} \cup \cdots$, with the edge $g G_{i}$ joining the vertex $g G_{i}$ to the vertex $g G_{i+1}$.) It follows that $G \in \mathbf{H}_{1} \mathfrak{F}$. By Proposition 3.6, $G$ cannot be in $\mathbf{H}_{1} \mathfrak{P}$.

Remark 3.9. Let $G$ be a group in $\mathbf{H} \mathfrak{F}$ that is also of type $\mathrm{FP}_{\infty}$. By a result of Kropholler [8], there is a bound on the orders of finite subgroups of $G$, and Kropholler-Mislin show that $G$ is in $\mathbf{H}_{1} \mathfrak{F}$ [9]. Corollary 3.2 shows that $G$ is in $\mathbf{H}_{1} \mathfrak{P}$. 


\section{Cohomology Relative to a family of Subgroups}

Let $\Delta$ denote a $G$-set, and let $\mathbb{Z} \Delta$ denote the corresponding $G$ module. For $\delta \in \Delta$, we write $G_{\delta}$ for the stabilizer of $\delta$. A short exact sequence $A \longmapsto B \rightarrow C$ of $G$-modules is said to be $\Delta$-split if and only if it splits as a sequence of $G_{\delta}$-modules for each $\delta \in \Delta$. Equivalently, the sequence is $\Delta$-split if and only if the following sequence of $\mathbb{Z} G$-modules splits: $A \otimes \mathbb{Z} \Delta \longmapsto B \otimes \mathbb{Z} \Delta \rightarrow C \otimes \mathbb{Z} \Delta[16]$.

We say a $G$ module is $\Delta$-projective if it is a direct summand of a $G$-module of the form $N \otimes \mathbb{Z} \Delta$, where $N$ is an arbitrary $G$-module. $\Delta$-projectives satisfy analogue properties to ordinary projectives. Furthermore, for each $\delta$, and each $G_{\delta}$-module $M$, the induced module $\operatorname{Ind}_{G_{\delta}}^{G} M$ is $\Delta$-projective. Given two $G$-sets $\Delta_{1}$ and $\Delta_{2}$ and a $G$-map $\Delta_{1} \rightarrow \Delta_{2}$ then $\Delta_{1}$-projectives are $\Delta_{2}$-projective and $\Delta_{2}$-split sequences are $\Delta_{1}$-split. For more detail the reader is referred to [16].

Now suppose that $\mathcal{F}$ is a family of subgroups of $G$ closed under conjugation and taking subgroups. We consider $G$-sets $\Delta$ satisfying the following condition:

$$
\Delta^{H} \neq \varnothing \Longleftrightarrow H \in \mathcal{F} \text {. }
$$

There are $G$-maps between any two $G$-sets satisfying condition (*), and so we may define an $\mathcal{F}$-projective module to be a $\Delta$-split module for any such $\Delta$. Similarly, an $\mathcal{F}$-split exact sequence of $G$-modules is defined to be a $\Delta$-split sequence. If $\Delta$ satisfies (*) and $M$ is any $G$-module, the module $M \otimes \mathbb{Z} \Delta$ is $\mathcal{F}$-projective and admits an $\mathcal{F}$-split surjection to $M$. This leads to a construction of homology relative to $\mathcal{F}$. An $\mathcal{F}$-projective resolution of a module $M$ is an $\mathcal{F}$-split exact sequence

$$
\cdots \rightarrow P_{n+1} \rightarrow P_{n} \rightarrow \cdots \rightarrow P_{0} \rightarrow M \rightarrow 0,
$$

where all $P_{i}$ are $\mathcal{F}$-projective. Group cohomology relative to $\mathcal{F}$, denoted $\mathcal{F} H^{*}(G ; N)$ can now be defined as the cohomology of the cochain complex $\operatorname{Hom}_{G}\left(P_{*}, N\right)$, where $P_{*}$ is an $\mathcal{F}$-projective resolution of $\mathbb{Z}$.

We say that a module $M$ is of type $\mathcal{F F P}$ if $M$ admits an $\mathcal{F}$ projective resolution in which $P_{i}$ is finitely generated for $0 \leq i \leq n$. It has been shown that modules of type $\mathcal{F F P}$ are of type $\mathrm{FP}_{n}$ [16]. We will say that a group $G$ is of type $\mathcal{F F P}$ if the trivial $G$-module $\mathbb{Z}$ is of type $\mathcal{F} \mathrm{FP}_{n}$.

We now specialize to the cases when $\mathcal{F}=\mathfrak{F}$ and $\mathcal{F}=\mathfrak{P}$.

Proposition 4.1. The following properties hold.

(i) A short exact sequence of $G$-modules is $\mathfrak{F}$-split if and only if it is $\mathfrak{P}$-split.

(ii) A G module is $\mathfrak{F}$-projective if and only if it is $\mathfrak{P}$-projective. 
(iii) $\mathfrak{F} H^{*}(G,-) \cong \mathfrak{P} H^{*}(G,-)$

Proof: (i) It is obvious that any $\mathfrak{F}$-split sequence is $\mathfrak{P}$-split, and the converse follows from a standard averaging argument. Let $H$ be an arbitrary finite subgroup of $G$. Then $|H|=\prod_{i=1, \ldots, n} p_{i}^{a_{i}}$ where $p_{i}$ are distinct primes and $0<a_{i} \in \mathbb{Z}$. For each $i$, let $n_{i}$ be the index $n_{i}=[H$ : $\left.P_{i}\right]$. Now consider a $\mathfrak{P}$-split surjection $A \stackrel{\pi}{\rightarrow} B$. Let $\sigma_{i}$ be a $P_{i}$-splitting of $\pi$, and define a map $s_{i}$ by summing $\sigma_{i}$ over the cosets of $P_{i}$ :

$$
s_{i}(b)=\sum_{t \in H / P_{i}} t \sigma_{i}\left(t^{-1} b\right) .
$$

For each $P_{i}$ we obtain a map $s_{i}: B \rightarrow A$, such that $\pi \circ s_{i}=n_{i} \times i d_{B}$. There exist $m_{i} \in \mathbb{Z}$ so that $\sum_{i} m_{i} n_{i}=1$, and the map $s=\sum_{i} m_{i} s_{i}$ is the required $H$-splitting.

(ii) It is obvious that a $\mathfrak{P}$-projective module is $\mathfrak{F}$-projective. Now let $P$ be $\mathfrak{F}$-projective. We may take a $\mathfrak{P}$-split surjection $M \rightarrow P$ with $M$ a $\mathfrak{P}$-projective. By (i) this surjection is $\mathfrak{F}$-split, and hence split. Thus $P$ is a direct summand of a $\mathfrak{P}$-projective and so is $\mathfrak{P}$-projective.

(iii) now follows directly from (i) and (ii).

Proposition 4.2. A group $G$ is of type $\mathfrak{F F P}_{0}$ if and only if $G$ has only finitely many conjugacy classes of subgroups of prime power order.

Proof: Suppose that $G$ has only finitely many conjugacy classes of subgroups in $\mathfrak{P}$. Let $I$ be a set of representatives for the conjugacy classes of $\mathfrak{P}$-subgroups and set

$$
\Delta_{0}=\bigsqcup_{P \in I} G / P
$$

This $G$-set satisfies condition $(*)$ for $\mathfrak{P}$ and therefore the surjection $\mathbb{Z} \Delta_{0} \rightarrow \mathbb{Z}$ is $\mathfrak{F}$-split and also $\mathbb{Z} \Delta_{0}$ is finitely generated.

To prove the converse we consider an arbitrary $\mathfrak{F}$-split surjection $P_{0} \rightarrow \mathbb{Z}$ with $P_{0}$ a finitely generated $\mathfrak{F}$-projective. As in $[16,6.1]$ we can show that $P_{0}$ is a direct summand of a module $\bigoplus_{\delta \in \Delta_{f}} \operatorname{Ind}_{G_{\delta}}^{G} P_{\delta}$, where $\Delta_{f}$ is a finite $G$-set, the $G_{\delta}$ are finite groups and $P_{\delta}$ are finitely generated $G_{\delta}$-modules. Therefore we might assume from now on that $P_{0}$ is of the above form. Since there is a $G$-map $\Delta_{f} \rightarrow \Delta$, where $\Delta$ satisfies condition $(*)$ the $\mathfrak{F}$-split surjection $P_{0} \stackrel{\varepsilon}{\rightarrow} \mathbb{Z}$ is also $\Delta_{f}$-split [16]. Consider now the following commutative diagram: 


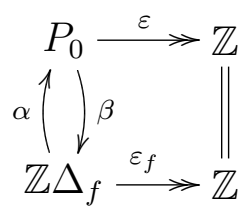

That we can find such an $\alpha$ follows from the fact that $\varepsilon$ is $\Delta_{f}$-split, and $\beta$ exists since $P_{0}$ is $\Delta_{f}$-projective being a direct sum of induced modules, induced from $G_{\delta},\left(\delta \in \Delta_{f}\right)$ to $G$.

As a next step we'll show that $\varepsilon_{f}$ is $\mathfrak{F}$-split. Take an arbitrary finite subgroup $H$ of $G$ and show that $\varepsilon_{f}$ splits when restricted to $H$. Since $\varepsilon$ is split by $s$, say, when restricted to $H$ we can define the required splitting by $\beta \circ s$.

Now let $P$ be an arbitrary $p$-subgroup of $G$. Since the module $\mathbb{Z}[G / P]$ is $\mathfrak{F}$-projective, there exists a $G$-map $\varphi$, such that the following diagram commutes:

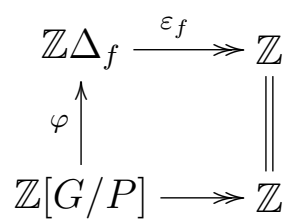

The image $\varphi(P)$ of the identity coset $P$ is a point of $\mathbb{Z} \Delta$ fixed by the action of $P$. If $H$ is any group and $\mathbb{Z} \Omega$ is any permutation module, then the $H$-fixed points are generated by the orbit sums $H$.w. Hence $P$ must stabilize some point of $\Delta_{f}$, since otherwise we would have that $p$ divides $\varepsilon_{f} \varphi(P)=\varepsilon \alpha(P)=1$, a contradiction. It follows that $P$ is a subgroup of $G_{\delta}$ for some $\delta \in \Delta_{f}$.

Note that being of type $\mathfrak{F} \mathrm{FP}_{0}$ does not imply that there are finitely many conjugacy classes of finite subgroups. In fact, the authors have examples with infinitely many conjugacy classes of finite subgroups, see [12]. Nevertheless this gives rise to the following conjecture:

Conjecture 4.3. A group $G$ is of type $\mathfrak{F F P}_{\infty}$ if and only if $G$ is of type $\mathrm{FP}_{\infty}$ and has finitely many conjugacy classes of p-subgroups.

It is shown in [16] that any $G$ of type $\mathfrak{F F P}_{\infty}$ is of type $\mathrm{FP}_{\infty}$, which together with Proposition 4.2 proves one implication in the above conjecture.

\section{REFERENCES}

[1] P. Abramenko and K. S. Brown, Buildings: Theory and Applications Graduate Texts in Mathematics 248, Springer (2008). 
[2] S. Bouc, Le complexe de chaînes d'un G-complexe simplicial acyclique, J. Algebra, 220, (1999), 415-436.

[3] G. E. Bredon, Introduction to compact transformation groups, Pure and Applied Math. 46, Academic Press (1972).

[4] K. S. Brown, Cohomology of groups Graduate Texts in Mathematics 87, Springer Verlag (1982).

[5] K. S. Brown, Buildings Springer (1989).

[6] D. Juan-Pineda and I. J. Leary. On classifying spaces for the family of virtually cyclic subgroups. Recent developments in algebraic topology. Contemporary Mathematics 407, 135-145 (2006).

[7] D. H. Kochloukova, C. Martínez-Pérez and B.E.A. Nucinkis, Cohomological finiteness conditions in Bredon cohomology, preprint 2008.

[8] P. H. Kropholler, On groups of type $F P_{\infty}$, J. Pure Appl. Algebra 90 (1993), $55-67$.

[9] P. H. Kropholler and G. Mislin, Groups acting on finite dimensional spaces with finite stabilizers, Comment. Math. Helv. 73 (1998), 122-136.

[10] P. H. Kropholler and C. T. C. Wall A theorem of Bouc concerning finite group actions on finite dimensional acyclic spaces, preprint.

[11] I. J. Leary, On finite subgroups of groups of type VF, Geom. Topol. 9 (2005), 1953-1976.

[12] I. J. Leary and B. E. A. Nucinkis, Some groups of type VF, Invent. math. 151 (2003), 135-165.

[13] W. Lück, The type of the classifying space for a family of subgroups, J. Pure Appl. Algebra 149 (2000), 177-203.

[14] W. Lück and M. Weiermann, On the classifying space of the family of virtually cyclic subgroups, arXiv:math.AT/0702646v1, 2007.

[15] C. Martinez-Pérez and B. E. A.Nucinkis, Cohomological Dimension of Mackey Functors for Infinite Groups, J. London Math. Soc. (2) 74 (2006), no. 2, 379396.

[16] B. E. A. Nucinkis, Cohomology relative to a G-set and finiteness conditions, Top. Appl. 92, (1999), 153-171.

[17] B. E. A. Nucinkis, Is there an easy algebraic characterisation of universal proper G-spaces?, manuscripta math. 102, (2000), 335-345.

[18] R. Oliver, Fixed-Point Sets of Group Actions on Finite Acyclic Complexes, Comment. Math. Helvetici 50 (1975), 155-177.

[19] D. Quillen, Homotopy properties of the poset of non-trivial p-subgroups of a group, Advances in Math. 28, (1978), 101-128.

[20] J. Tits, On buildings and their applications, Proc. Int. Cong. Mathematicians, Vancouver, 1974.

Department of Mathematics, The Ohio State University, $231 \mathrm{~W} 18 \mathrm{Th}$ Ave, Columbus Ohio 43210 
Heilbronn Institute, University of Bristol, Royal Fort Annexe, BRISTOL BS8 1TW

E-mail address: leary@math.ohio-state.edu

School of Mathematics, University of Southampton, Southampton SO17 1BJ, UK

E-mail address: B.E.A.Nucinkis@soton.ac.uk 